\title{
VIGENERE FOLLOWED BY MULTIPLE BIT-LEVEL PERMUTATIONS TO ENCRYPT A COLOR IMAGE
}

\author{
Abdellatif JarJar \\ Moulay Rachid High School, Taza Morocco IJCAT-326629
}

abdoujjar@gmail.com

\begin{abstract}
The largest part of the image encryption algorithms operates on the pixel as a central element by implementing diffusion confusion and eventually a permutation. On the other side, a permutation applied at the bit level changes not only the pixel value, but also its location within the image. In this work, we will propose a new technology of medical and color image encryption, based on chaotic permutations acting at the bit level, and a diffusion confusion ensured by an application of the method of Vigenere largely improved and adapted to the subject. Simulations performed on a large number of images of different sizes and formats ensure that our method is not subject to any known attacks.
\end{abstract}

\section{Article Highlights}

This new technology which applies Vigenere trick for protection against any differential attack followed by several permutations acting at bit level for color image encryption is based on the following novelties

$>$ New sequence Chaotic design

$>$ Implementation of Vigenere's advanced technique

$>$ Switching to binary writing

- Generation of chaotic permutations

- New method setup

$$
\text { Notation }\left\{\begin{array}{c}
G_{t}=\mathbb{Z} / t \mathbb{Z} \text { Ring } \\
A(: j): \text { Colunm } j \text { of matrix } A \\
A(j ;): \text { Line } j \text { of matrix } A \\
\oplus: \text { Xor operator } \\
E(x): \text { The whole part of } x
\end{array}\right.
$$

KEY WORD: VIGENERE GRID; CHAOTIC MAP; BROADCAST FUNCTION; CHAOTIC PERMUTATION;

I. INTRODUCTION

The rapid development of chaos theory in mathematics provides researchers with opportunities to further improve some classic encryption systems. In front of this great security focus, many techniques for color image 
encryption have flooded the digital world, mostly exploiting number theory and chaos By applying permutations at the pixel level [1-2-3-4-5]. Others are attempting to update their policies by improving some classical techniques, such as Hill [6-7-8], Cesar, Vignere [9-10], Feistel [11-12].

\section{1) Vigenere's classical technique}

This technology is based on static $(V)$ matrix defined by the following algorithm

algorithm $1\left\{\begin{array}{c}\text { Fist Row } \\ \text { For } i=1 \text { to } 26 \\ V(1, i)=i \\ \text { Next } i \\ \text { folloying Rows } \\ \text { For } i=2 \text { to } 26 \\ \text { For } j=1 \text { to } 26 \\ V(i, j)=V(i-1,(j+1), 26) \\ N \text { ext } j, i\end{array}\right.$

Let $(P)$ : plain text, $(C)$ : cypher text; $(K)$ : Encryption key, $(V)$ Vigenere matrix and $(l)$ : length of clear text. So

$$
\text { equation } 1\left\{\begin{array}{cc}
C_{i}=V\left(P_{i}, K_{i}\right)=\left(P_{i}+K_{i}\right) & \bmod 26 \\
P_{i}=V\left(C_{i}, K_{i}\right)=\left(P_{i}-K_{i}\right) & \bmod 26
\end{array}\right.
$$

Even though Vigenere's matrix was known, the encryption was able to withstand several centuries. But, Babagh's cryptanalysis is not efficient in not knowing the size of the encryption key. Several attempts to improve Vigenere's technique have invaded the digital world we quote [10 - 11]. In this work, the new structure of the substitution matrix and its attached replacement function will be described in detail.

\section{1) Our contribution}

Our contribution in this work is to start with a deeply improved trick of Vigenere for a suppression of any differential attack and to switch to binary notation to apply multiple permutations to encrypt a color image.

\section{THE PROPOSED METHOD}

Based on chaos [13-14-15], This new cryptosystem is based on the following axes

\section{AXE 1: CHAOTIC SEQUENCES DEVELOPMENT}

In order to build a new algorithm using a single-encryption key, we will use the $2 d$ logistics map [16-17-18].

This choice is due to the simplicity of its development and its high sensitivity to the initial parameters. 


\section{1) 2D Logistics Map Decryption}

It is a two-dimensional chaotic sequence defined by second degree polynomials. This sequence is given by the equation below

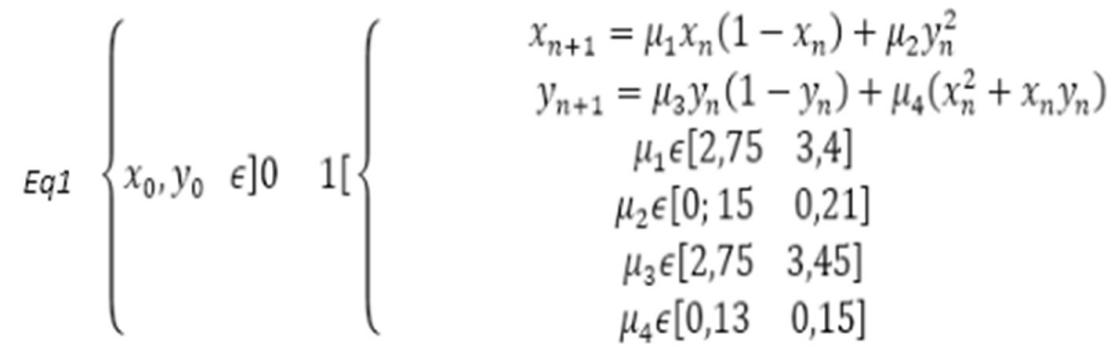

All the conditions ensure the installation of the chaotic aspect.

\section{2) Chaotic vector design}

Our work requires the construction of four chaotic vectors $(C L)$, $(C R),(K R)$ and $(K L)$ generated from the following algorithm

$\operatorname{Alg} 2\left\{\begin{array}{c}\text { Control and chaotic vector creation. } \\ \text { for } i=1 \text { to24nm } \\ C L(i)=\bmod \left(E\left(\frac{x(i)+2 y(i)}{3} * 10^{11}, 254\right)+1\right) \\ K L(i)=\bmod \left(E\left(\frac{x(i)+y(i) * y(i)}{2} * 10^{11}, 253\right)+2\right)\end{array} \quad\left\{\begin{array}{c}K R(i)=E\left(\frac{K L(i)+C L(i)}{2}\right) \\ \text { if } C L(i) \geq K L(i) \text { then } \\ C R(i)=0 \text { else } C R(i)=1 \\ \text { end if } \\ \text { Next } i\end{array}\right.\right.$

\section{3) Binary control vector development.}

we will construct two control vectors

$$
\text { Alg3 }\left\{\begin{array}{c}
\text { for } i=1 \text { to } 12 \mathrm{~nm} \\
\text { if } x(i) \geq y(i) \text { then } \\
B R(i)=0 \text { Else } \\
B R(i)=1 \\
\text { End if } \\
\text { Next } i
\end{array}\right.
$$

A passage of the $(C L)$ vector in matrix $(C M)$ of size $(8,3 \mathrm{~nm})$ is performed.

\section{AXE 2: VIGENERE UPGRADE}

In the first stage, Vigenere's [19-20 - 21] technology was greatly modified by integrating the new substitution matrix provided by the new powerful replacement function.

\section{1) Original Image Vectorization}

After the three $(R G B)$ color channels extraction and their conversion into size vectors $(\boldsymbol{V} \boldsymbol{r}),(\boldsymbol{V} \boldsymbol{g}),(\boldsymbol{V} \boldsymbol{b})(\mathbf{1}, \boldsymbol{n m})$ each, a concatenation is established to 
generate a vector $\boldsymbol{X}\left(\boldsymbol{x}_{\mathbf{1}}, \boldsymbol{x}_{2}, \ldots \ldots \ldots, \boldsymbol{x}_{\mathbf{3 n m}}\right)$ of size $(1,3 \mathrm{~nm})$. This operation is described by the following algorithm,

\section{a) Initialization value computation}

$$
\operatorname{Alg} 4\left\{\begin{array}{c}
\text { For } i=1 \text { to } n m \\
X(3 i-2)=V_{r}(i) \\
X(3 i-1)=V_{g}(i) \\
X(3 i)=V_{b}(i) \\
\text { Next } i
\end{array}\right.
$$

The initialization value must be calculated in order to change the startup pixel in the future and start the encryption process correctly.

The calculation of this value is described below

$$
\operatorname{Alg} 5\left\{\begin{array}{c}
\text { Initialization value computation. } \\
\text { for } i=2 \text { to } 3 \mathrm{~nm} \\
\text { If } C R(i)=0 \text { Then } \\
V=I V \oplus X(i) \oplus C L(i) \\
\text { Else } \\
I V=I V \oplus X(i) \oplus K L(i) \\
\text { End if } \\
\text { Next } i
\end{array}\right.
$$

The chaotic vector participates in the calculation of the initialization value mainly to avoid the problem of uniform image color.

\section{2) Vigenere's advanced methods}

This technique requires the establishment of two substitution matrices ( $V G)$ and $(V D)$ through the process described by the following steps

$\checkmark$ permutation $(R P)$ obtained by descending ordering the first 256 values of the sequence $(U)$

$\checkmark$ permutation (RR) obtained by increasing the ordering the first 256 values of the sequence $(V)$,

with the following restrictions

$$
\text { Eq2 }\left\{\begin{array}{l}
\text { If } P R(i)=256 \text { Then } P R(i)=0 \\
\text { If } R R(i)=256 \text { Then } R R(i)=0
\end{array}\right.
$$

This new construction is entirely supervised by the vector $(C R)$. It is given by the following algorithm 


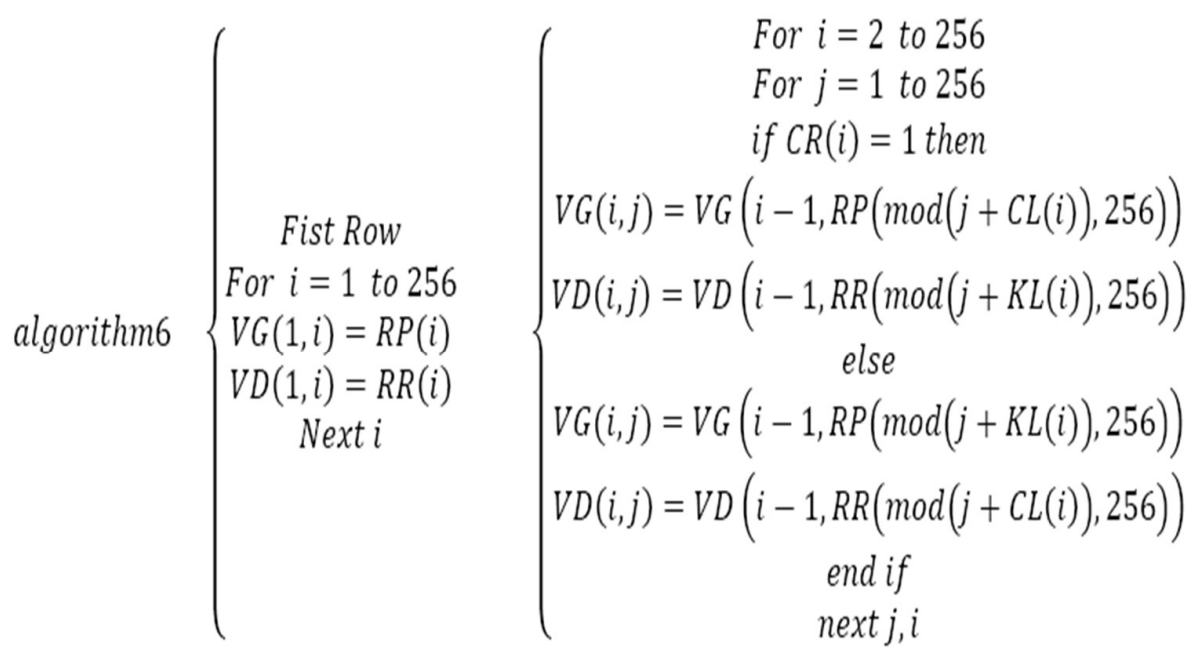

note that this building is completely done under the control of the vector $(C R)$.

Example: in $\left(\boldsymbol{G}_{8}\right)$

\begin{tabular}{|c|c|c|c|c|c|c|c|c|c|c|c|}
\hline$(V G)$ & 1 & 2 & 3 & 4 & 5 & 6 & 7 & 10 & $C R$ & $K L$ & $\mathrm{Cl}$ \\
\hline 1 & 3 & 5 & 0 & 6 & 2 & 7 & 1 & 4 & & & \\
\hline 2 & 2 & 77 & 1 & 4 & 3 & 5 & 0 & 6 & 1 & 5 & 4 \\
\hline 3 & $T^{\top}$ & 3 & 5 & 0 & 6 & 2 & 7 & 1 & 1 & 3 & 5 \\
\hline 4 & 2 & 7 & 1 & 4 & 3 & 5 & 0 & 6 & 0 & 3 & 4 \\
\hline 5 & 0 & & 2 & 7 & 1 & 4 & 3 & . & 1 & 4 & 2 \\
\hline
\end{tabular}

\begin{tabular}{|l|l|l|l|l|l|l|l|l|}
\hline$(V D)$ & 1 & 2 & 3 & 4 & 5 & 6 & 7 & 0 \\
\hline 1 & 0 & 4 & 5 & 7 & 1 & 2 & 6 & 3 \\
\hline 2 & 7 & 1 & 2 & 3 & 3 & 0 & 4 & 5 \\
\hline 3 & 0 & 4 & 5 & 7 & 1 & 2 & 6 & 3 \\
\hline 4 & 1 & 2 & 6 & 3 & 0 & 4 & 5 & 7 \\
\hline 5 & 0 & 4 & 5 & 7 & 1 & 2 & 6 & 3 \\
\hline
\end{tabular}

a) New Vigenere's mathematical expression

Based on Vigenere's classic formula, given by the following formula

$$
\text { Eq3 } Y(i)=V G(C L(i), X(i))
$$

In this work the image $Y(i)$ of pixel $X(i)$ is given by the formula below

\section{2) First Encryption Process}

$$
E q 4\left\{\begin{array}{c}
\text { If } B R(i)=0 \text { Then } \\
V_{1}(X(i))=Y(i)=V G(C L(i), V D(K L(i), X(i) \oplus K R(i))) \\
\quad E l s e \\
V_{2}(X(i))=Y(i)=V D(K L(i), V G(K R(i), X(i) \oplus C L(i)))
\end{array}\right.
$$

The figure below illustrates the first encryption process improved using only Vigenere 


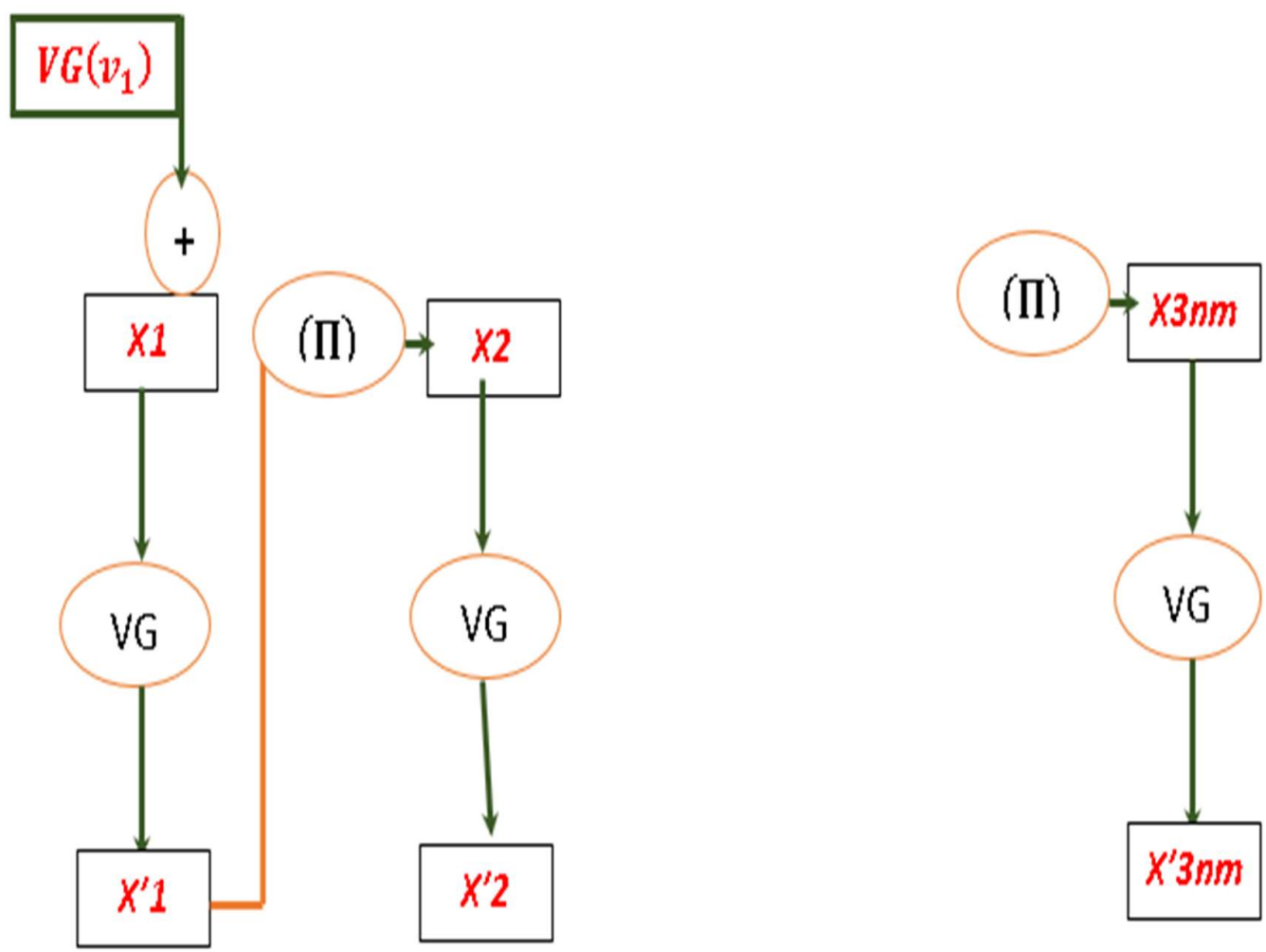

Figure2 : First Encryption

$\{\quad(V)$ : advanced substitution matrix

(П): New diffusion function

(II) The new diffusion, defined by the following equation

$$
\text { Eq5 } \Pi(X(i+1))=V D\left(K R(i), V G\left(C L(i), X^{\prime}(i) \oplus X(i+1)\right)\right)
$$

This function links the encrypted pixel with the next clear pixel. This schema is translated by the algorithm below

$$
\operatorname{Alg} 7\left\{\begin{array}{c}
X^{\prime}(1)=V G(K R(1), V D(C L(1), I V \oplus X(1))) \\
\text { For } i) 2 \text { to } 3 n m \\
\Pi(X(i))=V D\left(K R(i), V G\left(C L(i), X^{\prime}(i-1) \oplus X(i)\right)\right) \\
I f B R(i)=0 \text { Then } \\
X^{\prime}(i)=V_{1}(X(i)) \\
\text { Else } \\
X^{\prime}(i)=V_{2}(X(i)) \\
\text { End if } \\
\text { Next } i
\end{array}\right.
$$

We note that this first step uses only substitutions, which ensures an extreme speed in the execution. The output vector $\boldsymbol{X}^{\prime}\left(\boldsymbol{x}_{1}^{\prime}, \boldsymbol{x}_{2}^{\prime}, \ldots \ldots, \boldsymbol{x}_{\mathbf{3 n m}}^{\prime}\right)$, 'will undergo a second encryption attempt. 


\section{1) First round analysis}

For a better follow-up of our algorithm, several reference images were tested by this first round, we quote

\section{Example: "Lena" encrypted in the first round}
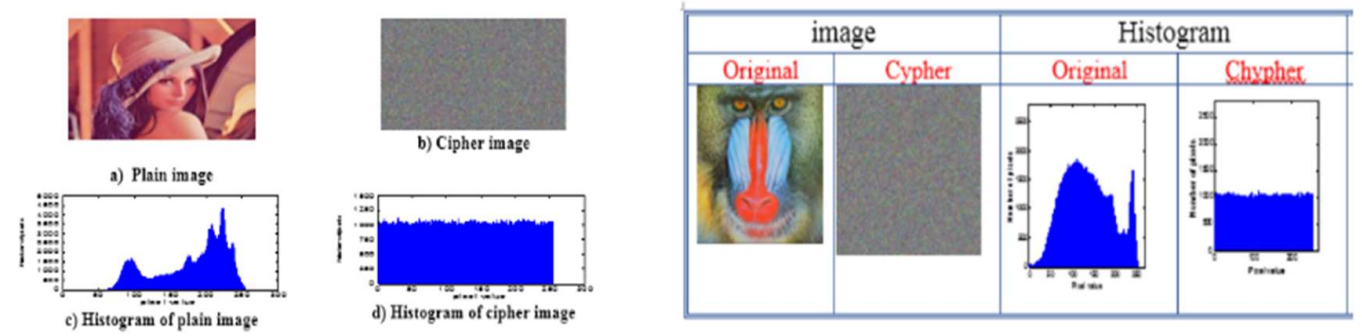

\section{AXIS 4: PERMUTATION GENERATION}

Eight permutations $\left(Q_{i}\right)_{1}^{8}$ are generated by the following process [22 - $23-$ 24]

$\left(Q_{i}\right)$ permutation in $\left(G_{3 n}\right)$ obtained by a descending sort in the broad sense on the row $(i)$ of the matrix $(C M)$

\section{1) Binary Writing}

The vector $\left(X^{\prime}\right)$ is converted into binary to obtain a matrix $(X M)$ of size $(8.3 \mathrm{~nm})$. This process follows the following path

$$
\left\{\begin{array} { c } 
{ \text { Switching to hexadecimal } } \\
{ x = E ( \frac { X ( i ) } { 1 6 } ) } \\
{ y = X ( i ) - 1 6 * x }
\end{array} \quad \left\{\begin{array}{c}
\text { Passage in } G_{4} \\
\alpha=E\left(\frac{x}{4}\right) \\
\beta=x-4 \alpha \\
\gamma=E\left(\frac{x}{4}\right) \\
\delta=y-4 \gamma
\end{array}\right.\right.
$$

We consider the table $(T)$ of binary values lower than 4

\begin{tabular}{|l|l|l|}
\hline$(T)$ & 1 & 2 \\
\hline 0 & 0 & 0 \\
\hline 1 & 0 & 1 \\
\hline 2 & 1 & 0 \\
\hline 3 & 1 & 1 \\
\hline
\end{tabular}

Finally, the passage of the vector $\left(X^{\prime}\right)$ in binary matrix $(X M)$ is defined by the following algorithm 


$$
\left\{\begin{array}{c}
\text { For } i=1 \text { to } 3 \text { nm } \\
x=E\left(\frac{X^{\prime}(i)}{16}\right) \\
y=X^{\prime}(i)-16 * x \\
\alpha=E\left(\frac{x}{4}\right) \\
\beta=x-4 * \alpha \\
\gamma=E\left(\frac{y}{4}\right) \\
\delta=y-4 * \gamma \\
X M(1, i)=T(\alpha, 1) \\
X M(2, i)=T(\alpha, 2) \\
X M(3, i)=T(\beta, 1) \\
X M(4, i)=T(\beta, 2) \\
X M(5, i)=T(\gamma, 1) \\
X M(6, i)=T(\gamma, 2) \\
X M(7, i)=T(\gamma, 1) \\
X M(8, i)=T(\gamma, 2) \\
N \text { ext } i
\end{array}\right.
$$

Each row $(i)$ of the matrix $(X M)$ is subjected to the permutation $\left(Q_{i}\right)_{1}^{8}$, the resulting matrix $(M X)$ is given by the algorithm below

$$
\operatorname{Alg} 9\left\{\begin{array}{c}
\text { For } i=1 \text { to } 8 \\
\text { For } j=1 \text { to } 3 \mathrm{~nm} \\
M X(i, j)=X M\left(i, Q_{i}(j)\right) \\
\text { Next } j, i
\end{array}\right.
$$

Example:

Permutations

\begin{tabular}{|l|l|l|l|l|l|l|l|l|}
\hline & $\left(Q_{1}\right)$ & $\left(Q_{2}\right)$ & $\left(Q_{3}\right)$ & $\left(Q_{4}\right)$ & $\left(Q_{5}\right)$ & $\left(Q_{6}\right)$ & $\left(Q_{7}\right)$ & $\left(Q_{8}\right)$ \\
\hline 1 & 3 & 5 & 3 & 2 & 3 & 5 & 4 & 6 \\
\hline 2 & 2 & 3 & 2 & 3 & 1 & 6 & 6 & 1 \\
\hline 3 & 5 & 6 & 4 & 5 & 5 & 2 & 3 & 3 \\
\hline 4 & 1 & 4 & 6 & 4 & 6 & 1 & 5 & 5 \\
\hline 5 & 4 & 1 & 5 & 1 & 4 & 4 & 1 & 2 \\
\hline 6 & 6 & 2 & 1 & 6 & 2 & 3 & 2 & 4 \\
\hline
\end{tabular}

Clear Pixel

\begin{tabular}{|l|l|l|l|l|l|l|}
\hline & $Y(1)$ & $Y(2)$ & $Y(3)$ & $Y(4)$ & $Y(5)$ & $Y(6)$ \\
\hline 1 & 1 & 0 & 0 & 1 & 1 & 0 \\
\hline 2 & 0 & 0 & 1 & 1 & 1 & 0 \\
\hline 3 & 0 & 1 & 1 & 1 & 1 & 0 \\
\hline 4 & 0 & 0 & 1 & 0 & 1 & 0 \\
\hline 5 & 1 & 0 & 0 & 0 & 0 & 1 \\
\hline 6 & 0 & 1 & 1 & 0 & 0 & 1 \\
\hline 7 & 1 & 0 & 0 & 0 & 1 & 1 \\
\hline 8 & 1 & 0 & 1 & 1 & 0 & 1 \\
\hline & 130 & 36 & 117 & 224 & 141 & 15 \\
\hline
\end{tabular}

\begin{tabular}{|l|l|l|l|l|l|l|}
\hline & $X(1)$ & $X(2)$ & $X(3)$ & $X(4)$ & $X(5)$ & $X(6)$ \\
\hline & 209 & 42 & 140 & 226 & 49 & 101 \\
\hline $\mathbf{1}$ & 1 & 0 & 1 & 1 & 0 & 0 \\
\hline $\mathbf{2}$ & 1 & 0 & 0 & 1 & 0 & 1 \\
\hline $\mathbf{3}$ & 0 & 1 & 0 & 1 & 1 & 1 \\
\hline $\mathbf{4}$ & 1 & 0 & 0 & 0 & 1 & 0 \\
\hline $\mathbf{5}$ & 0 & 1 & 1 & 0 & 0 & 0 \\
\hline 6 & 0 & 1 & 1 & 0 & 0 & 1 \\
\hline $\mathbf{7}$ & 1 & 1 & 0 & 1 & 0 & 0 \\
\hline $\mathbf{8}$ & 0 & 0 & 1 & 1 & 1 & 1 \\
\hline
\end{tabular}

Cypher Pixel 


\section{STEP 5: DECR YPTION ENCR YPTED IMAGES}

The solid chaining establishes between the encrypted block and the next clear block in the broadcast process, forcing us to start decryption from the last block using the opposite functions. So, the decryption process should follow these steps

- Binary conversion

- Reciprocal permutation generation

- Switching to grayscale

- Vigenere reciprocal matrix

- Vigenere's reciprocal application

\section{1) Reciprocal permutation generation}

Consider $\left(T_{i}\right)_{1}^{8}$ the reciprocal permutation of $\left(Q_{i}\right)_{1}^{8}$, it defines by the following algorithm

2) Vigenere Reciprocal matrix

$$
\operatorname{Alg} 10\left\{\begin{array}{c}
\text { For } j=1 \text { to } 3 n m \\
T_{i}\left(Q_{i}(j)\right)=j \\
\text { Next } j
\end{array}\right.
$$

$$
\operatorname{Alg} 11\left\{\begin{array}{c}
\text { for } i=1 \text { to } 256 \\
\text { for } j=1 \text { to } 256 \\
G V(i, V G(i, j))=j \\
D V((i, V D(i, j))=j \\
N \text { ext } j, i
\end{array}\right.
$$

\begin{tabular}{|c|c|c|c|c|c|c|c|c|c|c|c|c|c|c|c|c|c|c|c|}
\hline (VG) & 1 & 2 & 3 & 4 & & 7 & 0 & $C R$ & $K L$ & $C L$ & (GV) & 1 & 2 & 3 & 4 & 5 & 6 & 7 & 0 \\
\hline 1 & 3 & 5 & 0 & 5 & 27 & 1 & 4 & & & & 1 & 7 & 5 & 1 & 0 & 2 & 4 & 6 & 3 \\
\hline 2 & 2 & 7 & 1 & 4 & 35 & 0 & 6 & 1 & 5 & 4 & 2 & 3 & 1 & 5 & 4 & 6 & 0 & 2 & 7 \\
\hline 3 & 4 & 3 & 5 & 5 & 52 & 7 & 1 & 1 & 3 & 5 & 3 & 0 & 6 & 2 & 1 & 3 & 5 & 7 & 4 \\
\hline 4 & 2 & 7 & 1 & 4 & 35 & 0 & 61 & 0 & 3 & 4 & 4 & 3 & 1 & 5 & 4 & 6 & 0 & 2 & 7 \\
\hline 5 & 0 & & 2 & 7. & & 13 & 5 & 1 & 4 & 2 & 5 & 5 & 3 & 7 & 6 & 0 & 2 & 4 & 1 \\
\hline
\end{tabular}

\section{Example}

By following the same logic of Vigenere's traditional technique, we obtain

$$
\text { Equation6 }\left\{\begin{array}{c}
\text { if } z=V G(y, x) \\
\text { Then } \\
x=G V(y, z)
\end{array}\right.
$$


3) Vigenere's inverse expression

4) Reverse diffusion

$$
\text { Algo12 }\left\{\begin{array}{c}
\text { if } B R(k)=0 \text { then } \\
V_{1}^{-1}\left(X^{\prime}{ }_{k}\right)=D V\left(K L(k), G V\left(C L(k), X^{\prime}{ }_{k}\right)\right) \oplus K R(k) \\
\text { else } \\
V_{2}^{-1}\left(X^{\prime}{ }_{k}\right)==G V\left(K R(k), D V\left(K L(k), X^{\prime}{ }_{k}\right)\right) \oplus C L(k) \\
\text { end if }
\end{array}\right.
$$

Equation $22 \quad \Pi^{-1}\left(X^{\prime}(k)\right)=\mathrm{GV}\left(C L(k), D V\left(K R(k), X^{\prime}(\mathrm{k})\right) \oplus X(k-1)\right.$

\section{AXIS 5: EXAMPLES AND SIMULATIONS}

In order to measure the performance of our encryption system, we randomly select a large number of reference images, and then use our method to test them

\section{1) Brutal assaults}

They consist in reconstructing the encryption keys in a random manner.

\section{a. Key-space analysis}

The chaotic sequence used in our method ensures strong sensitivity to initial conditions, and can protect it from any brutal attacks.

The secret key to our system consists of

$$
\text { Equation } 7 \quad \begin{cases}x_{0}=0,7655412001, & \mu_{1}=3.89541 \\ y_{0}=0.865421331, & \mu_{2}=0,56120 \\ \mu_{3}=1,3561 & \mu_{4}=0,56321\end{cases}
$$

If we use single-precision real numbers $\mathbf{1 0}^{-\mathbf{1 0}}$ to operate, the total size of the key will greatly exceed $\approx \mathbf{2}^{\mathbf{1 8 0}} \gg \mathbf{2}^{\mathbf{1 1 0}}$, which is enough to avoid any brutal attacks.

\section{b. Secret key's sensitivity Analysis}

Our encryption key has a high sensitivity, which means that a small degradation of a single parameter used will automatically cause a large difference from the original image. This ensures that in the absence of the real encryption key, the original image cannot be restored.

\section{2) Histogram analysis}

all images tested by our algorithm have a uniformly distributed histogram. This reflects that the entropy of the encrypted images is around 8, which makes the system immune to histogram attacks. The tablel shows that the horizontal correlation values of the encrypted images are close to zero. This ensures high security against correlation attacks. 
Table 1: Encrypted image histogram

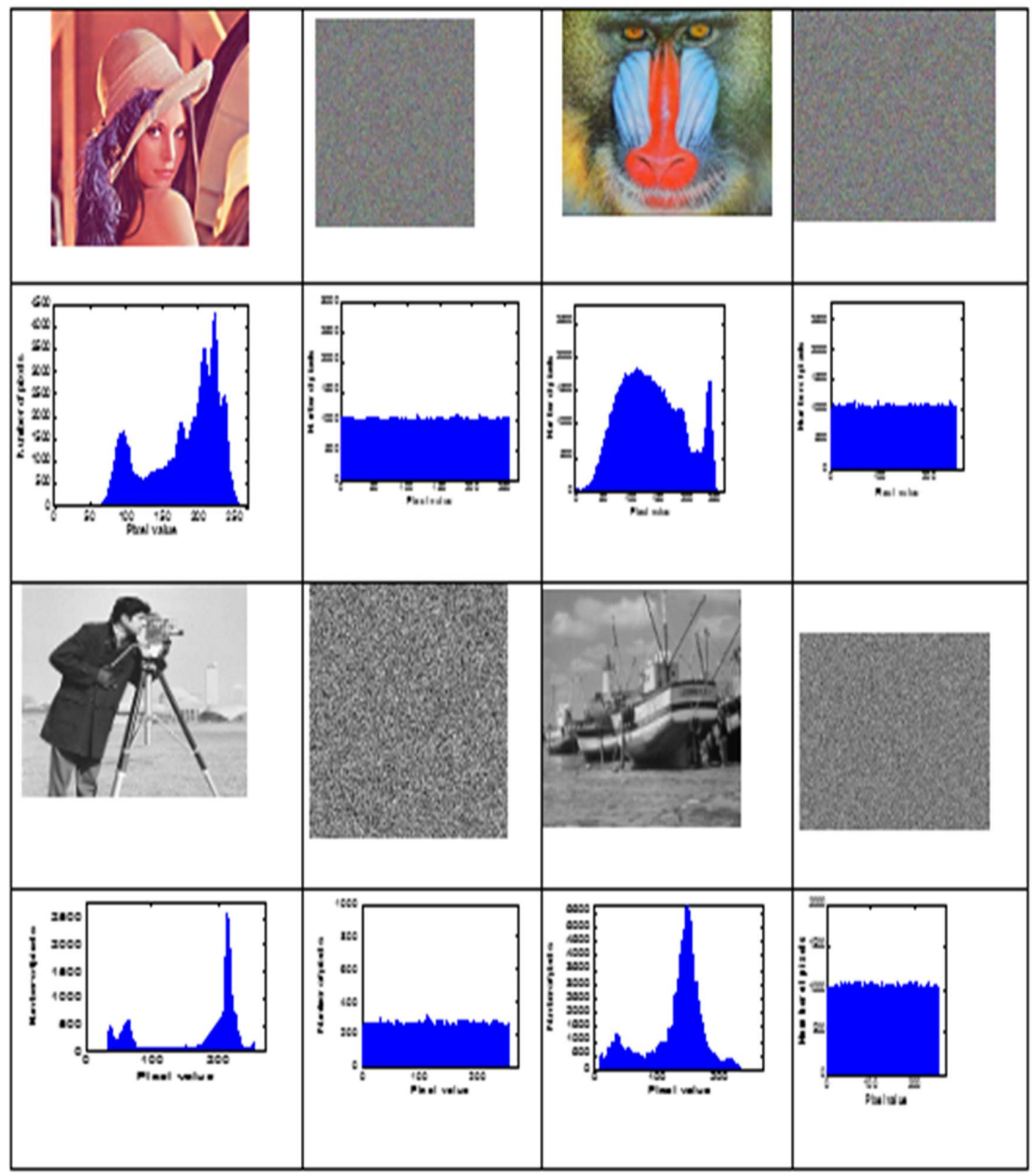

\section{3) Statistics Attack Security}

\section{a. Entropy Analysis}

Entropy is the measure of the disorder diffused by a source without memory. The entropy expression is determined by the equation below

$$
\text { Equation } 8 \quad H(M C)=\frac{1}{t} \sum_{i=1}^{t}-p(i) \log _{2}(p(i))
$$

The entropy values on the 150 images arbitrarily chosen from a large database of images of different sizes and formats, tested by our method are represented graphically by the following figure 


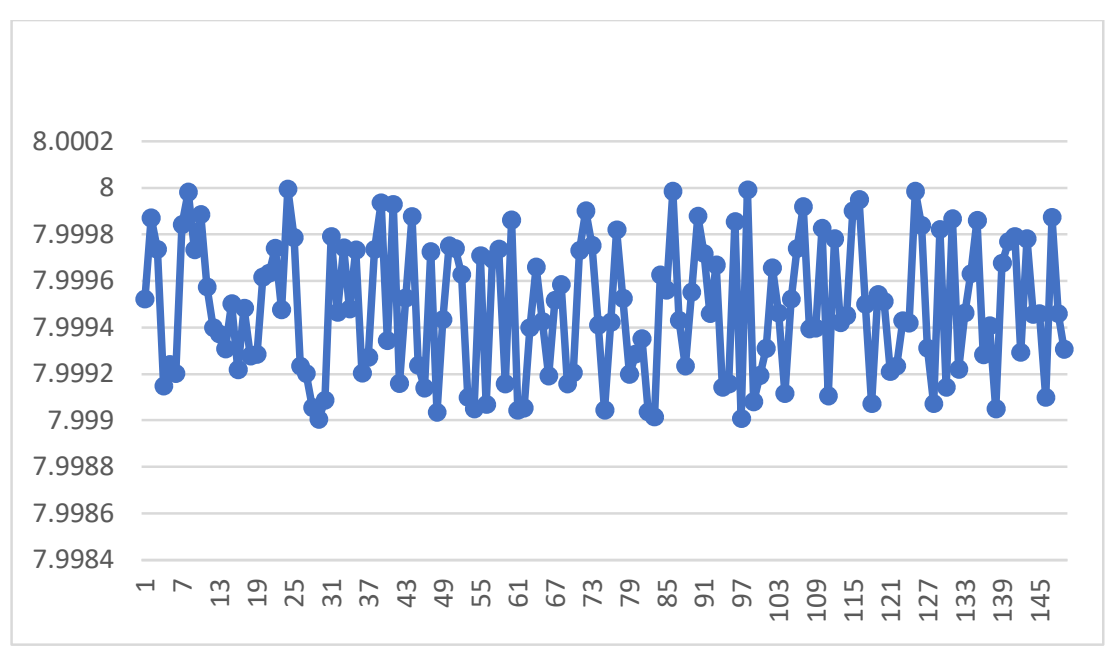

Fugure3: Entropy of 150 images

All the entropy values of the images tested by our algorithm are close to 8, which confirms the uniformity of the histogram. This proves that the method is far from a statistical attack.

\section{b. Entropy statistical analysis}

We will study the uniformity of the distribution of entropy released by the test.

\section{i. Position parameter analysis}

The values derived from the entropy by applying our approach to over 150 images in our image database, constitute a statistical series with position, dispersion and concentration parameters have been recalculated to verify the safety of our approach.

The purpose of this analysis is to show that the distribution follows a reduced central normal distribution. So

$$
\text { Equation } 9\left\{\begin{array}{l}
Q_{1}=\text { First quartile } \\
Q_{2}=\text { Second quartile } \\
Q_{3}=\text { Third quartile }
\end{array}\right.
$$

Position Parameters

\begin{tabular}{|l|l|l|l|l|l|}
\hline Average & Max & Min & Q1 & Q2 & Q3 \\
\hline 7,999120 & 7,99993210 & 7,999002 & 7,9990132 & 7,999310 & 7,999721 \\
\hline
\end{tabular}

\section{c. Correlation analysis}

Correlation is a technique that compares two images to estimate the displacement of pixels in one image relative to another reference image. The relevant expression is defined by the following equation 
equation 29 correlation $\quad r=\frac{\operatorname{cov}(x, y)}{\sqrt{V(x)} \sqrt{V(y)}}$

\section{i. Horizontal Correlation}

The following figure graphically represents the simulation of 150 color images of the same size, which are selected from an image database of various sizes, formats and related values

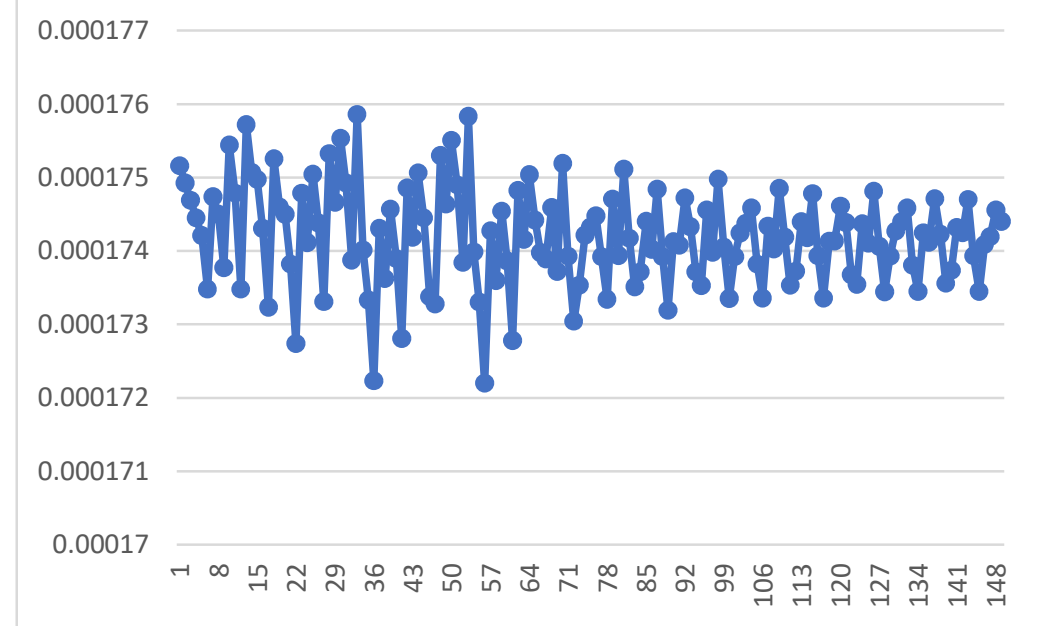

Fugure4: Entropy of 150 images

\begin{tabular}{|c|c|c|c|c|c|}
\hline Average & Max & Min & Q1 & Q2 & Q3 \\
\hline 7,999120 & 0,000179 & 0,000170 & 0,000173 & 0,000174 & 0,000176 \\
\hline
\end{tabular}

\section{ii. Vertical Correlation}

Simulations made on 150 images of the database gave the vertical correlation scores are displayed in Figure below

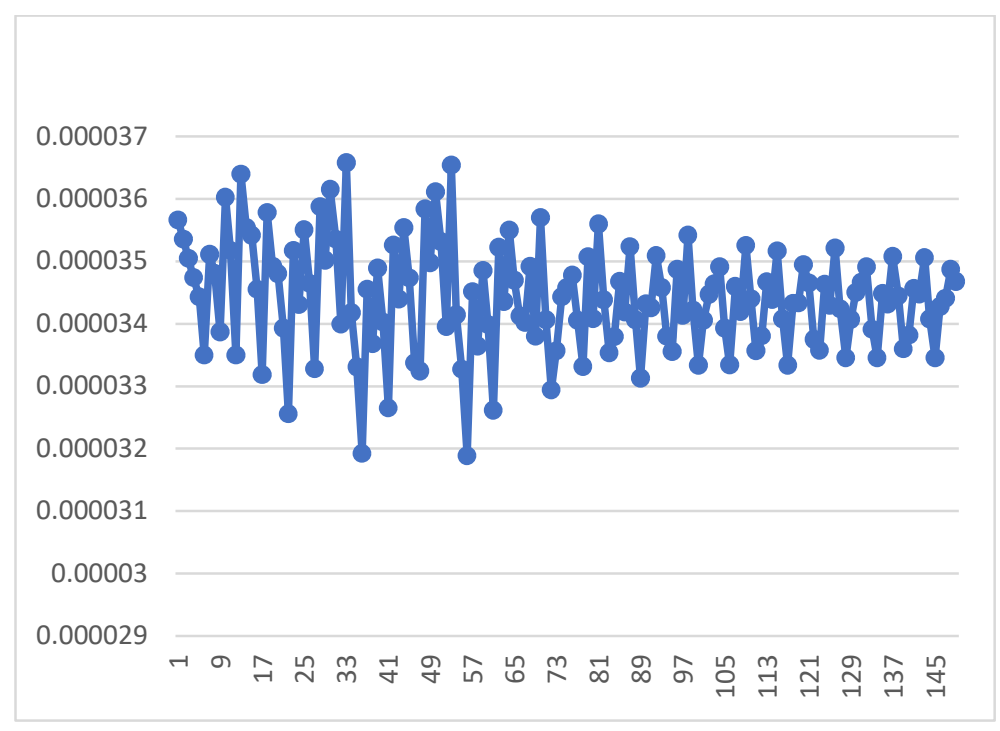

flgure5: Vertical correlation of 150 images 
Figure 5 shows that the vertical correlation values of the encrypted images are close to zero. This ensures high security against correlation attacks.

\section{iii. Diagonal Correlation}

Simulations made on 150 images of the database gave the diagonal correlation scores are displayed in Figure below

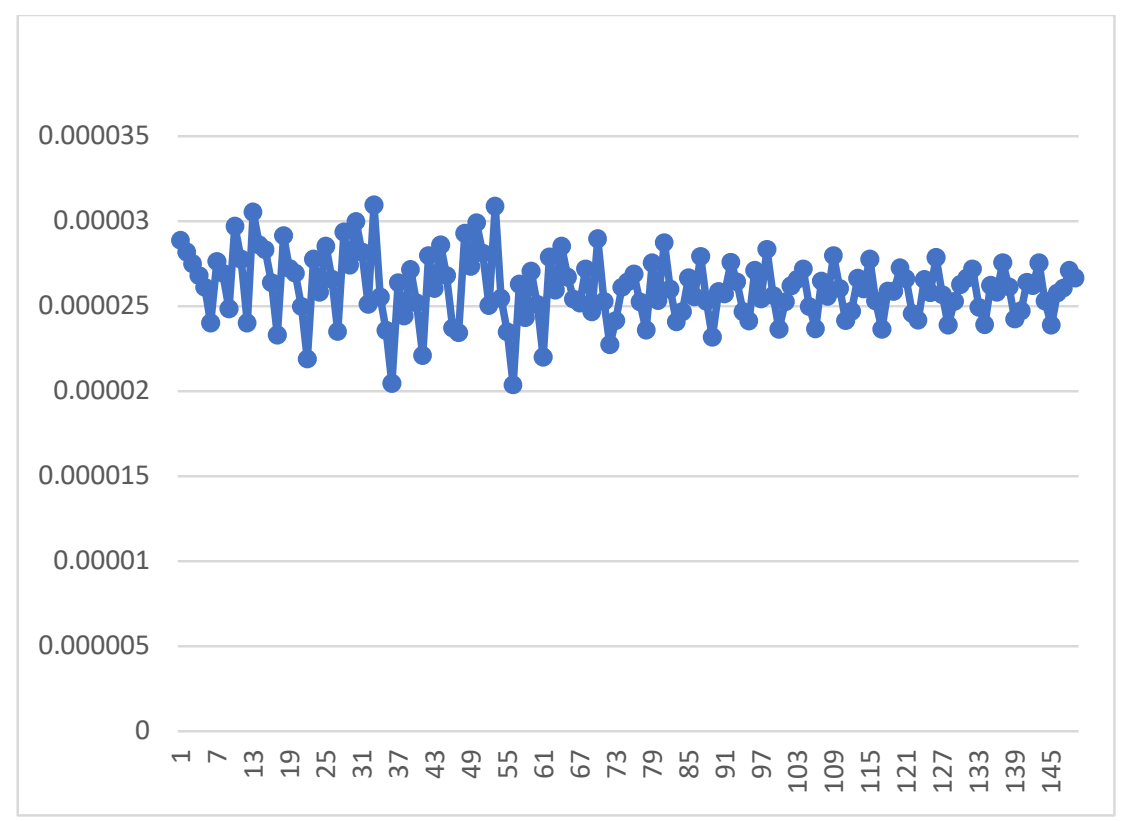

flgure6: Diagonal correlation of 150 images

Figure above shows that the diagonal correlation values of the encrypted images are close to zero. This ensures high security against correlation attacks.

\section{4) Differential analysis}

Let be two encrypted images, whose corresponding free-to-air images differ by only one pixel, from $\left(\boldsymbol{C}_{\mathbf{1}}\right)$ and $\left(\boldsymbol{C}_{\mathbf{2}}\right)$, respectively. The $N P C R$ mathematical analysis of an image is given by the equation below

$$
\begin{gathered}
\text { Equation } 10 \quad N P C R=\left(\frac{1}{n m} \sum_{i, j=1}^{n m} D(i, j)\right) * 100 \\
\text { With } D(i, j)=\left\{\begin{array}{lll}
1 & \text { if } & C_{1}(i, j) \neq C_{2}(i, j) \\
0 & \text { if } & C_{1}(i, j)=C_{2}(i, j)
\end{array}\right.
\end{gathered}
$$

The UACI mathematical analysis of an image is given by the below

$$
\text { Equation } 11 \quad U A C I=\left(\frac{1}{n m} \sum_{i, j=1}^{n m} \operatorname{Abs}\left(C_{1}(i, j)-C_{2}(i, j)\right)\right) * 100
$$

The study of the 150 selected images revealed the following diagram 


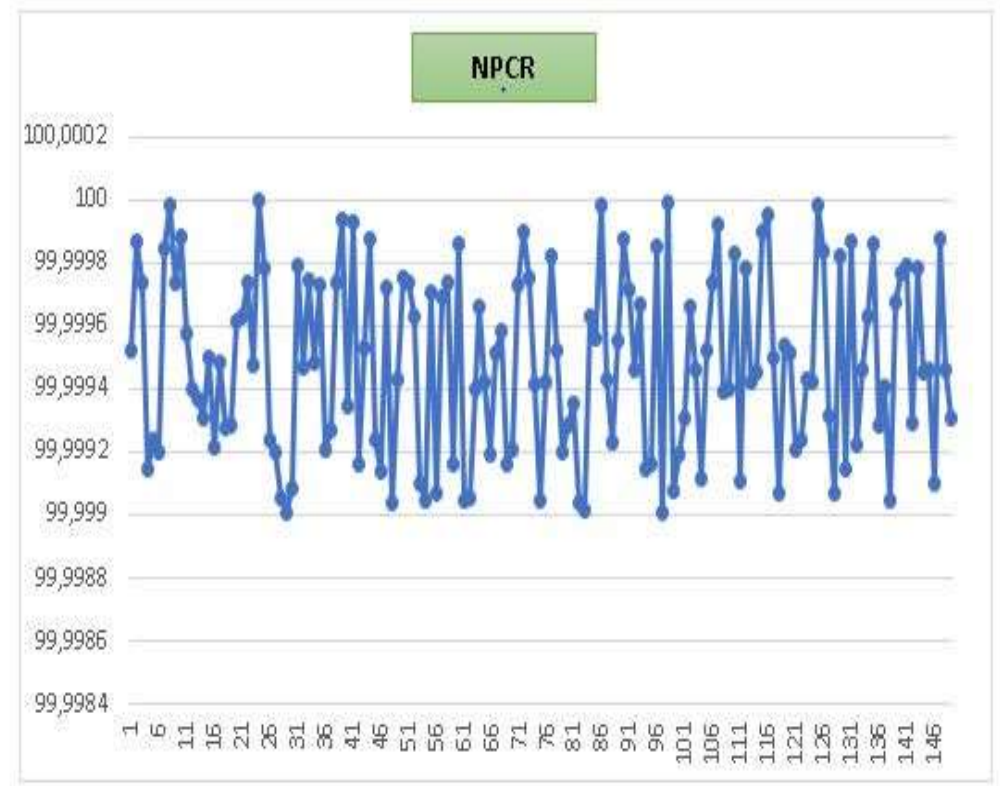

flgure7: NPCR of 150 images

\begin{tabular}{|l|l|l|l|l|l|}
\hline Average & Max & Min & Q1 & Q2 & Q3 \\
\hline 99,995 & 99,999 & 99,991 & 99,993 & 99,994 & 99,997 \\
\hline
\end{tabular}

All detected values are inside the confidence interval [99,63 99,95]. These values are largely sufficient to affirm that our crypto system is protected from known differential attacks

The study of the 150 selected images revealed the following diagram

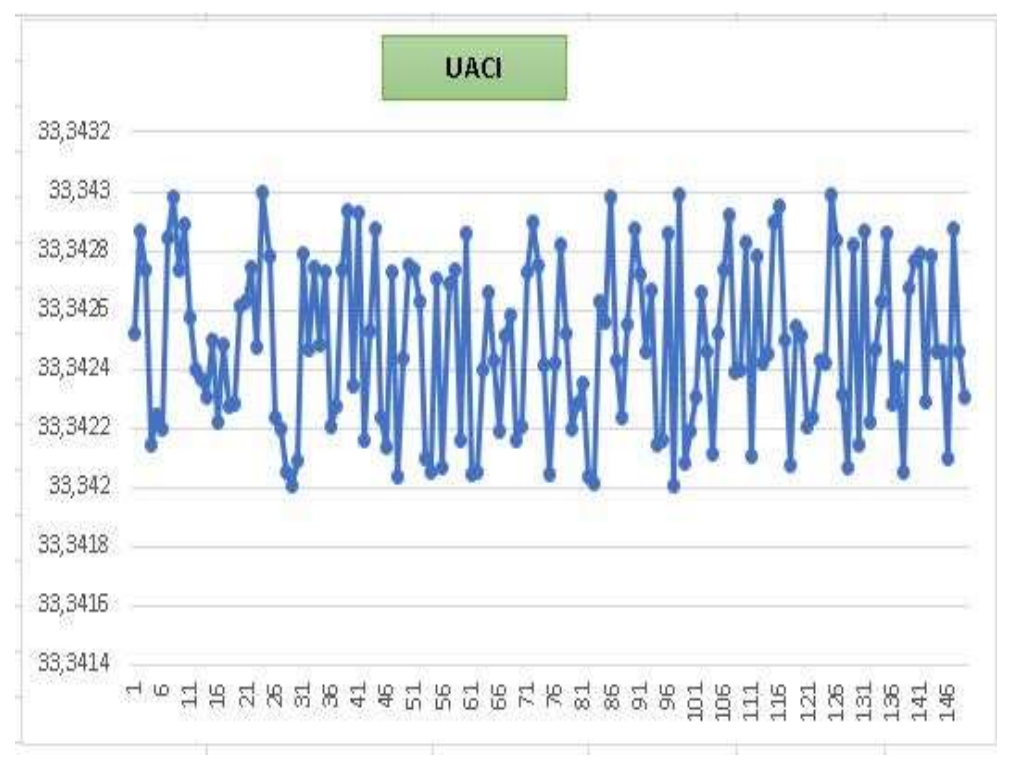

\section{flgure8: UACl of 150 images}

All detected values are inside the confidence interval [33;34 33,35]. These values are largely sufficient to affirm that our crypto system is protected from known differential attacks. 


\section{5) Avalanche effect}

The avalanche effect is a required property in virtually all cryptographic hash functions and block coding algorithms. It causes progressively more important changes as the data is propagating in the structure of the algorithm. This constant determines the avalanche impact of the cryptographic structure in place. It is approximated by the equation below

$$
\text { Equation } 12 A E=\left(\frac{\sum_{i} \text { bit change }}{\sum_{i} \text { bit total }}\right) * 100
$$

Figure below depicts the evaluation of the $A E$ score for 150 images examined by our approach.

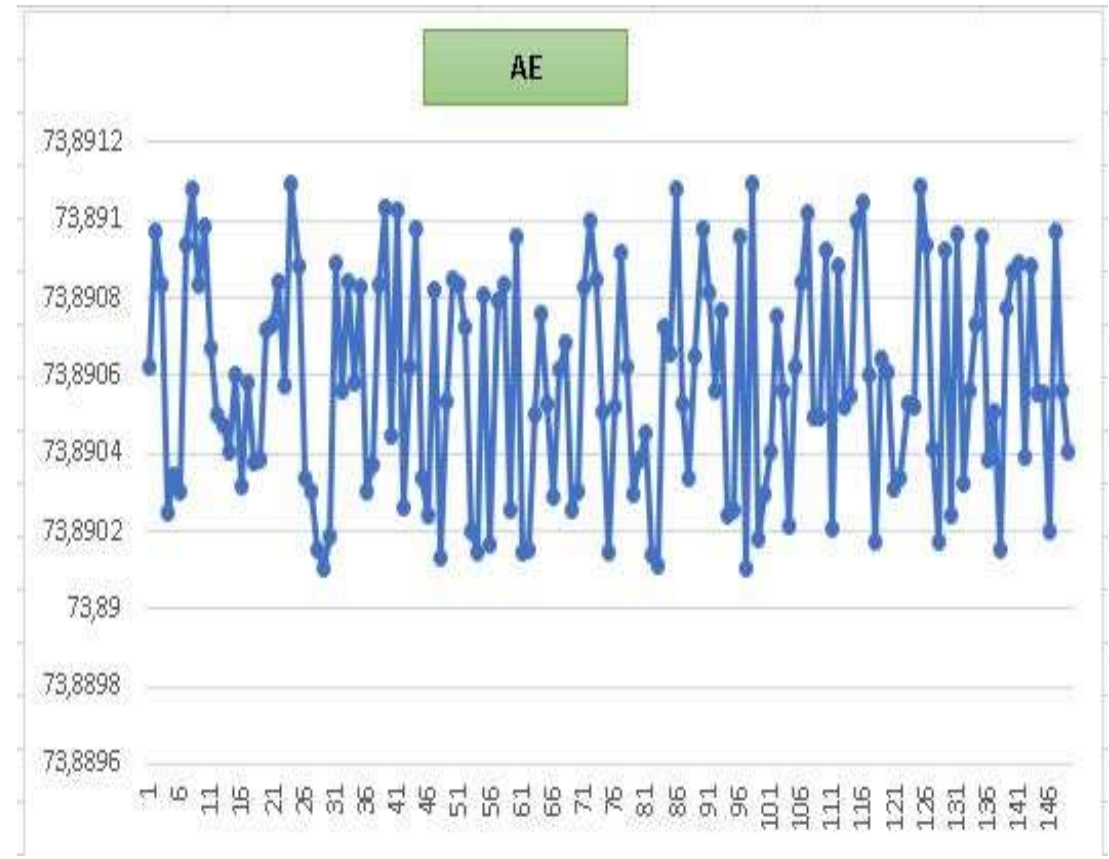

Figure9: Avalanche effect

\begin{tabular}{|c|c|c|c|c|c|}
\hline Average & Max & Min & Q1 & Q2 & Q3 \\
\hline 73,8906 & 73,892 & 73,890 & 73,8903 & 73,8905 & 73,891 \\
\hline
\end{tabular}

All values returned from the $(A E)$ by our method are all in the range of residual values $[73,8973,892]$. This guarantees that a one- bit change in the clear image will be reflected by a change of at least $78 \%$ of the encrypted image's bits.

\section{6) Signal-To-Peak Noise Ratio (PSNR)}

\section{(a) $M S E$}

The image quality estimation to be based on the pixel change was obtained by processing the (PSNR) values and the (MSE). It is calculated by the following equation 


$$
\text { Equation } 13 \quad M S E=\sum_{i, j}(P(i, j)-C(i, j))^{2}
$$

$(P(i, j))$; pixel of the clear image

$(C(i, j))$ : pixel of the cypher image

(b) PSNR

The signal-to-peak noise ratio, often abbreviated PSNR, is an engineering term for the ratio between a signal's maximum possible power and the power of distorted noise that affects the precision of its display. The PSNR mathematical analysis of an image is given by the next equation

$$
\text { Equation } 14 P S N R=20 \log _{10}\left(\frac{I_{\max }}{\sqrt{M S E}}\right)
$$

For $(R G B)$ color images, the definition of (PSNR) is the same except that the (MSE) is the sum of all square value changes. In the alternative, for color images, the image is transcoded into a separate color space and the PSNR is displayed for each channel in that color space.

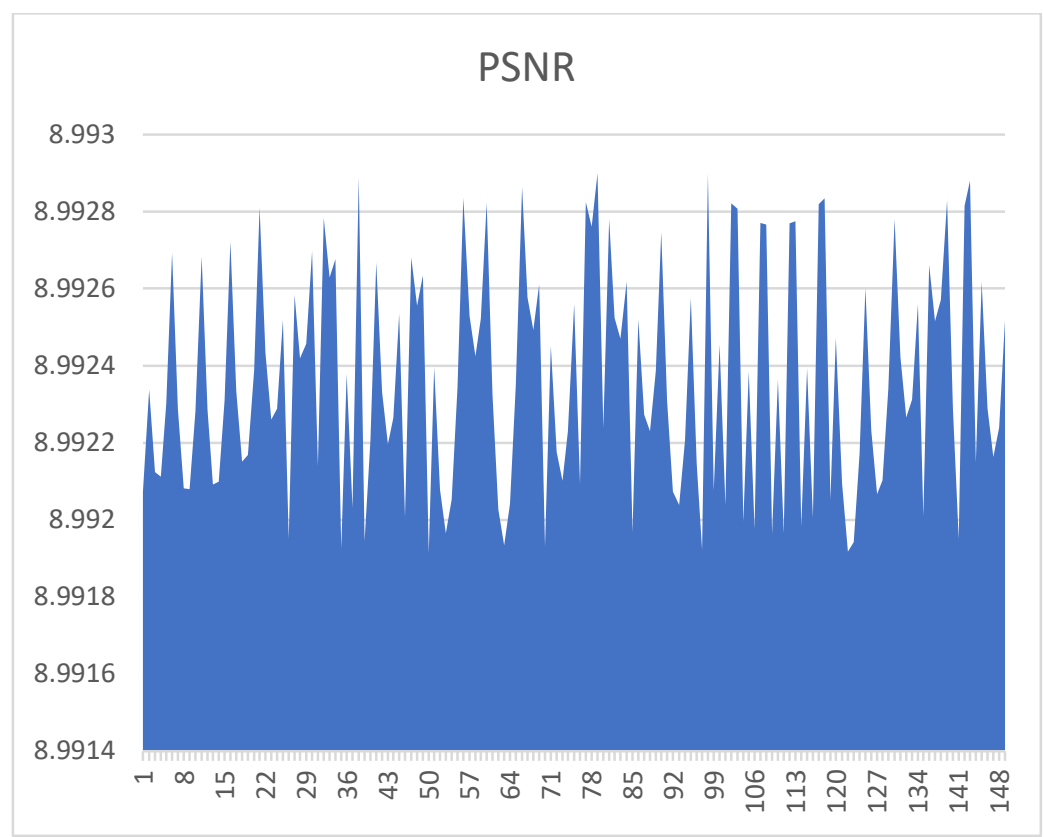

flgure10: "PSNR of 150 images

All values returned from the (PSNR) by our method are all in the range of residual values [8,99 8,993].

\section{b) Speed analysis}

For an evaluation of the execution time, our algorithm is tested on a personal computer "Intel core i5 3337 U 1.86 Gz CPU 8 GB ram. We use Matlab as programming software. We measure the encryption and decryption time of the tested images. 


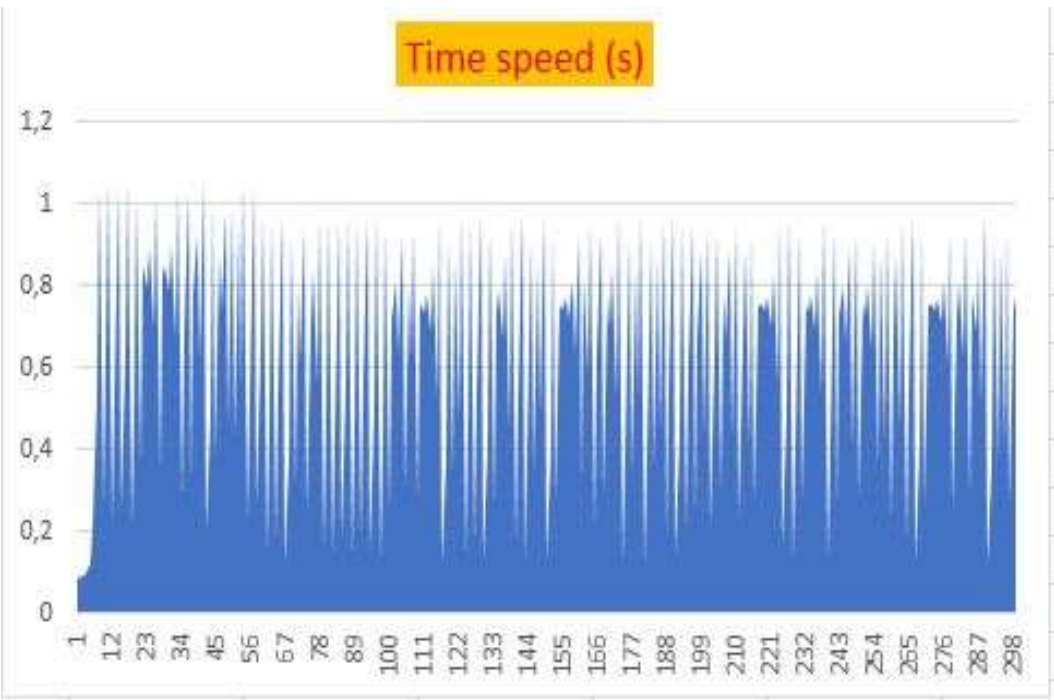

flgure11: time of 300 images

II. MATH SECURITY

The size of the encryption key protects the system from brute force attacks. The construction of the chaotic permutations is difficult to reverse. Moreover, the established chaining protects the algorithm from differential attacks.

III. CONCLUSION

This new algorithm which starts with an implementation of the greatly improved Vigenere technique ensured by two chaotic substitution matrices and installation of a diffusion ensured also by the two Vigenere matrices, followed by a passage in binary writing for an application of eight chaotic permutations acting at the level of the bits for a better diffusion confusion can face any known attack .

\section{References}

\begin{tabular}{|r|l|}
\hline [1] & $\begin{array}{l}\text { Saiyma Fatima Raza « A novel bit permutation-based image encryption algorithm » Nonlinear } \\
\text { Dynamic 95, pages859-873(2019) }\end{array}$ \\
\hline [2] & $\begin{array}{l}\frac{\text { Guangfeng Cheng, Chunhua Wang » A Novel Color Image Encryption Algorithm Based on }}{\text { Hyperchaotic System and Permutation-Diffusion Architecture “International Journal of Bifurcation }} \\
\text { and Chaos Vol. 29, No. 09, 1950115 (2019) }\end{array}$ \\
\hline [3] & $\begin{array}{l}\text { RasulEnayatifar "Index-based permutation-diffusion in multiple-image encryption using DNA } \\
\text { sequence "Optics and Lasers in Engineering Volume 115, April 2019, Pages 131-140 }\end{array}$ \\
\hline [4] & $\begin{array}{l}\text { LinqingHuang « On symmetric color image encryption system with permutation-diffusion } \\
\text { simultaneous operation "Optics and Lasers in Engineering Volume 115, April 2019, Pages 7-20 }\end{array}$ \\
\hline [5] & $\begin{array}{l}\text { Shamama Anwa "A pixel permutation based image encryption technique using chaotic map } \\
\text { "Multimedia Tools and Applications volume 78, pages27569-27590(2019) }\end{array}$ \\
\hline
\end{tabular}




\begin{tabular}{|c|c|}
\hline [6] & $\begin{array}{l}\text { A.P.USiahaan "Genetic algorithm in Hill cipher encryption"international association of scientific } \\
\text { innovation and research (IASR) vol 15,no,12016 }\end{array}$ \\
\hline [7] & A.S alkhalid " cryptanalyse of Hill cipher using genetic algorithm" dalam IEEE hanmument 2015 \\
\hline [8] & $\begin{array}{l}\text { A.Jarjar « Improvement of hill' sclassical method in image cryptography »International Journal of } \\
\text { Statistics and Applied Mathematics } 2017 \text {, Volume } 2 \text { Issue 3, Part A }\end{array}$ \\
\hline [9]. & $\begin{array}{l}\text { Imam Saputra, Mesran, Nelly Astuti Hasibuan3, "Vigenere Cipher Algorithm with Grayscale Image } \\
\text { Key Generator for Secure Text File" International Journal of Engineering Research \& Technology } \\
\text { (IJERT), Vol. } 6 \text { Issue 01, January-2017 }\end{array}$ \\
\hline [10]. & $\begin{array}{l}\text { Vaka Vamshi Krishna Reddy, Sreedhar Bhukya 2, "ENCRYPT AND DECRYPT IMAGE USING VIGENERE } \\
\text { CIPHER", International Journal of Pure and Applied Mathematics, Volume } 118 \text { No. } 242018\end{array}$ \\
\hline [11] & $\begin{array}{l}\text { A.P.U siahaan" three pass protocol in Hill cipher encryption technique" international journal of } \\
\text { science and research (IJSR) vol } 5 \text { nà } 72016 \text { pp 1149-1152 }\end{array}$ \\
\hline [12] & $\begin{array}{l}\text { I Gede Arya Putra Dewangga, Tito Waluyo Purboyo, Ratna Astuti Nugrahaeni," A New Approach of } \\
\text { Data Hiding in BMP Image Using LSB Steganography and Caesar Vigenere Cipher Cryptography" } \\
\text { International Journal of Applied Engineering Research, Volume 12, Number } 21 \text { (2017) pp. 10626- } \\
\underline{10636}\end{array}$ \\
\hline [13] & $\begin{array}{l}\text { Overbey.J.traversW and W ydylo J } 2005 \text { " On the key space of the Hill cipher" Cryptologia 29(1), 59- } \\
\underline{72}\end{array}$ \\
\hline [14] & Saeednia,S 2000 "haow to make the Hill secure" Cryptologia 24(2), 353-360 \\
\hline [15] & $\begin{array}{l}\text { Lin.C.H.Lee.C.Y and Lee.C.Yu } 2004 " \text { comments on saeednia's improved scheme for the Hill cipher." } \\
\text { Journal of the chineese institute of engineers } 27 / 5,743-746\end{array}$ \\
\hline$[16]$ & $\begin{array}{l}\text { A.Jarjar « Improvement of hill's classical method in image cryptography » International Journal of } \\
\text { Statistics and Applied Mathematics 2017, Volume } 2 \text { Issue 3, Part A }\end{array}$ \\
\hline [17] & $\begin{array}{l}\text { IYongWang all » A chaos-based image encryption algorithm with variable control parameters" } \\
\text { Chaos, Solitons \& Fractals Volume 41, Issue 4, } 30 \text { August } 2009 \text {, Pages } 1773-1783^{\prime \prime}\end{array}$ \\
\hline [18] & $\begin{array}{l}\text { Jan Sher Khan all, "Chaos based efficient selective image encryption" Multidimensional Systems and } \\
\text { Signal Processing volume 30, pages943-961(2019)" }\end{array}$ \\
\hline [19] & $\begin{array}{l}\text { H Li, Y Wang, Z Zuo - Optics and Lasers in Engineering, } 2019 \text { "Chaos-based image } \\
\text { encryption algorithm with orbit perturbation and dynamic state variable selection mechanisms" } \\
\text { Volume 115, April 2019, Pages 197-207 }\end{array}$ \\
\hline [20] & $\begin{array}{l}\text { Rongjun Ge all «A Novel Chaos-Based Symmetric Image Encryption Using Bit-Pair Level Process" } \\
\text { July 8, 2019, date of current version August 7, } 2019 .\end{array}$ \\
\hline [21] & $\begin{array}{l}\text { Mohamed JarJar "Further improvement of the HILL method applied in image encryption" Procedia } \\
\text { computer sciences 00(2019)000-000 }\end{array}$ \\
\hline [22] & $\begin{array}{l}\text { Shams Mahmoud Abd Ali » Novel Encryption Algorithm for Securing Sensitive Information Based on } \\
\text { Feistel Cipher"Test engeenering managenment Page Number: } 10-16 \text { Publication Issue:19 Volume: } \\
\underline{80 \text { September-October } 2019}\end{array}$ \\
\hline$[23]$ & $\begin{array}{l}\text { Zhi-hua Gan » A chaotic image encryption algorithm based on 3-D bit-plane permutation » Neural } \\
\text { Computing and Applications (2019) 31:7111-7130 }\end{array}$ \\
\hline
\end{tabular}

